\title{
Nonlinear constitutive models for pultruded FRP composites
}

\author{
Rami Haj-Ali *, Hakan Kilic \\ School of Civil and Environmental Engineering, Georgia Institute of Technology, Atlanta, GA 30332-0355, USA
}

Received 20 December 2001; received in revised form 19 April 2002

\begin{abstract}
Three nonlinear multi-axial constitutive models for pultruded fiber reinforced plastic composites are proposed and examined in this study. The first two are macromechanical models that idealize the entire composite material as homogeneous orthotropic under plane stress conditions. The third is a new three-dimensional (3D) micromechanical model where the fiber and matrix responses are explicitly recognized and the nonlinear behavior is expressed at the matrix level. The pultruded composite material system considered in this study consists of two alternating layers of roving and continuous filament mat. The two layers have E-glass/vinylester fiber/matrix constituents. Coupon tests were performed for calibration and verification of the proposed models. Nonlinear response is calibrated using V-notch tests, to generate the axial-shear stress-strain, and uniaxial transverse tests. Off-axis coupons were cut with different roving orientations in order to generate in-plane multi-axial stress states. The nonlinear axial stress-strain curves of the off-axis tests are compared with the predicted curves from the three proposed models. Good agreement is shown for all off-axis angles when comparing the experimental stress-strain curves with those predicted by the 3D micromodel. The nonlinear curves predicted by the two nonlinear orthotropic models are also in good agreement with the experimental results but not for all off-axis orientations. All three models can be easily integrated within a finite element code for the general nonlinear analysis of pultruded composite structures using layered shell or 3D type elements.
\end{abstract}

(c) 2002 Elsevier Science Ltd. All rights reserved.

Keywords: Nonlinear; Stress-strain; Micromechanics; Pultruded; Off-axis; Composite

\section{Introduction}

Pultrusion is a manufacturing process where structural members having a constant crosssection are made with different reinforcement systems: unidirectional bundles of fibers (roving), woven, and nonwoven mats, such as continuous

\footnotetext{
${ }^{*}$ Corresponding author. Tel.: +1-404-894-4716; fax: +1-404894-0211.

E-mail addresses: rami.haj-ali@ce.gatech.edu, gt8061b@ prism.gatech.edu (R. Haj-Ali).
}

filament mat (CFM). Pultruded members can have a relatively thick cross-section with a typical thickness range of $1 / 4^{\prime \prime}-1^{\prime \prime}$ and a combination of reinforcements. Unlike laminated composites, pultruded composites are usually thicker and can have the same volume as traditional metallic structural profiles. Therefore, pultruded composites are favorable candidates to be used for high strength and light weight civil and infrastructure applications. There are additional advantages of thick-section pultruded composites, such as being corrosion resistant and electrical or magnetic insulators in transmission towers. 
Pultruded fiber reinforced plastic (FRP) composites may exhibit nonlinear stress-strain behavior under multi-axial static loading conditions. This nonlinearity usually results from the behavior of the soft polymeric matrix constituent while the fibers (roving) generally exhibit elastic and brittle behavior. The presence of manufacturing defects in the form of voids and microcracks amplifies this nonlinear material response. Geometric discontinuities at the structural level, such as cracks, holes, and cutouts, enhance this behavior. The nonlinear behavior can influence the overall structural response and its mode of failure especially at higher load levels. Therefore, nonlinear material modeling in the analysis of pultruded structures can be important in order to determine accurate states of deformations.

Many experimental and analytical studies have been focused on the nonlinear response of laminated composite materials. Macrolevel theories are formulated to characterize the nonlinear response by idealizing the composite as anisotropic homogeneous medium. Petit and Waddoups (1969) proposed an incremental approach for the analysis of symmetric laminates under uniform membrane loading. In their approach, the elastic constants of a lamina are determined as a function of the lamina strains. A lamina failure is identified when any of its strain components exceeds the experimentally obtained limiting strain value. Once a failure mode is detected in the lamina, the corresponding tangent stiffness is set to a high negative value in order to achieve stress unloading in this direction. Their analyses predicted the overall shape of the nonlinear response quite well, while the laminate ultimate failure loads were predicted with acceptable accuracy.

Hahn and Tsai (1973) used the complementary energy density polynomial function, for a lamina under a plane-stress state, to derive a nonlinear stress-strain relation for laminated composites. An additional fourth-order term of the axial-shear stress is added to the polynomial function. This term represents the nonlinear shear strain. Interaction terms between the three stress components were neglected. Hahn (1973) extended this work to model the nonlinear behavior of laminates, and considered the effect of material nonlinearity on the buckling load of a symmetric laminate. Hashin et al. (1974) formulated a new nonlinear constitutive model where inelastic transverse and axial shear strains exist in a lamina under plane stress conditions. Each inelastic strain component is an independent quadratic function in terms of the stress invariants raised to a general power. Ramberg-Osgood representation of nonlinear stressstrain curves was used to express the nonlinear axial-shear and transverse stress-strain relations. Predicted nonlinear behavior compared well with experimental results for different laminated composite materials.

Jones and Nelson (1974) proposed a material model based on strain energy that accounts for nonlinear behavior under biaxial states of stresses. This model can only be applied to multi-axial stress states where the strain energy is lower than the maximum strain energy generated in the uniaxial test results. Since this is often the case when the composite is subject to general multi-axial stress states, different approaches were proposed to overcome this difficulty, Jones and Morgan (1977), Abu-Farsakh (1989).

Amijima and Adachi (1979) introduced a simple technique to represent the shear nonlinearity using piecewise linear segments. Sandhu (1976) introduced a technique for solution of the nonlinear laminate equations using piecewise cubic spline functions to represent the lamina uniaxial test data. $\mathrm{He}$ also proposed a predictor-corrector iterative method. Nahas (1984) presented a similar predictor-corrector technique in which the basic uniaxial stress-strain relations are allowed to have a general nonlinear representation. Kuppuswamy et al. (1984) used the Richard and Abbott (1975) representation for the nonlinear uniaxial stress-strain relations in a three-dimensional finite element analysis. However, their study uses uncoupled onedimensional stress-strain relations which do not reflect interaction effects. Pindera and Herakovich (1983) and Mathison et al. (1991) derived a nonlinear plane stress constitutive model using orthotropic endochronic theory. This theory is based on irreversible thermodynamics with internal variables.

Dvorak and Rao (1976) proposed a plasticity theory for fibrous composites under axisymmetric 
deformation. Plastic dilatation and deformation of the composite in the fiber direction were accounted for. A yield function was formed using the stress invariants of a transversely isotropic material and simple hardening and flow rules were derived. Griffin (1981) extended Hill's anisotropic plasticity theory, and used the associated plasticity flow rule to determine the plastic increment of strain. A Ramberg-Osgood uniaxial stress-strain relation was used to model nonlinear hardening. Sun and Chen (1989) developed a one parameter orthotropic plasticity model for thermoplastic and metal-matrix composite laminates in plane stress. In this model, one-parameter plastic-potential scalar function is proposed. Only transverse and axial shear stresses are involved in the plastic potential; therefore, plastic deformation in the axial direction of the lamina is neglected. In addition, a power law relation between effective plastic strain and the effective stress is proposed; as a result, a total of three material parameters are needed to fully describe the lamina plastic behavior.

The above nonlinear constitutive models have been mainly applied to laminated composites under plane stress conditions. These models have not yet been applied to account for the nonlinear behavior of pultruded composites. In fact, multiaxial nonlinear models for pultruded composites have been limited. This can be attributed to the fact that pultruded composite structural members are designed to carry predominant axial loading and the unidirectional roving is the main reinforcement that is often used. As a result, constant axial properties and linear material response are usually combined with beam or plate theories to perform general structural analysis. Luciano and Barbero (1994) proposed models that can predict the overall initial stiffness from micromechanical theories for each composite system (layer) that forms the cross-section of a pultruded member. Classical lamination theory and mechanics of laminated beams were used to predict the overall axial stiffness. Haj-Ali et al. (2001) have recently proposed a three-dimensional (3D) micromechanics-based framework for linear and nonlinear analysis of pultruded composite materials and structures. This material and structural framework integrates different nonlinear 3D micromechanical models for each of the reinforcement layers that form the pultruded cross-section. Each micromodel recognizes the linear or nonlinear response of the fiber and matrix constituents.

This study presents the nonlinear 3D micromechanical material models used for the roving and CFM layers of a pultruded section. The Hahn and Tsai (1973) and Hashin et al. (1974) homogeneous orthotropic (plane stress) macromodels are also implemented and examined, along with the $3 \mathrm{D}$ micromodels, in their ability to predict the nonlinear multi-axial response of E-glass/vinylester pultruded composite material. The experimental results of Haj-Ali and Kilic (2002) are used to calibrate the linear and nonlinear material properties needed in the three proposed models. Off-axis coupons under compression are used to examine the nonlinear prediction of the above constitutive models. These coupons make it possible to examine the nonlinear material response for different in-plane stress states.

\section{Pultruded composite material system}

The composite coupons tested in this study were cut from a $1 / 2^{\prime \prime}$ pultruded plate with alternating layers of unidirectional roving and CFM. This plate was manufactured by Creative Pultrusion Inc. and designated as series 1625 material system. The fibers in the CFM are relatively long, swirl, and randomly oriented in the plane. The roving layers consist of unidirectional fiber bundles that run in the pultrusion direction along the entire span of the member. The CFM and roving layers can have different thicknesses through the crosssection, depending on the level of reinforcement and number of mats used. The fibers in the roving and the CFM in the tested plate are both made from E-glass material. The matrix is vinylester and it is mixed with small clay particles. This matrix medium, defined herein as the effective medium of the vinylester polymer, clay particles, and voids or microcracks, is considered as a nonlinear isotropic material. The fiber volume fractions (FVFs) of the unidirectional roving and the CFM were determined by a series of burn-out tests. The average FVFs are 0.407 for the roving and 0.305 for the 
CFM. These are average FVF values calculated by assuming a uniform thickness of all CFM or roving layers. The relative average thickness of the CFM layers is $(0.328 / 0.5)$, while the relative thickness for the roving is $(0.172 / 0.5)$. The combined average FVF in the pultruded material, i.e. in both the roving and CFM volumes, is 0.34. There is an apparent number of void systems spread inside the pultruded section as reported by Haj-Ali et al. (2001). The existence of voids in laminated FRP composites was reported in several previous studies (Binshan et al., 1995; Wang and Zureick, 1994). Moreover, the roving reinforcement in pultruded materials can be unevenly distributed which can lead to a reduction in stiffness and strength (Herakovich and Mirzadeh, 1991). Haj-Ali and Kilic (2002) showed that an E-glass/ vinylester pultruded material system has a lower initial elastic modulus in tension than the corresponding compressive modulus. Also, the nonlinear response of the pultruded material is softer in tension than the behavior in compression.

\section{Micromechanical models for the roving and CFM layers}

Nonlinear constitutive models are proposed in this study in order to generate the through-thickness effective stress-strain response at different locations of the pultruded structural member. Two approaches are viable. The first recognizes the nonlinear behavior of each layer within the thickness and applies separate micromechanical models that are able to generate the $3 \mathrm{D}$ nonlinear response. The second approach is to use constitutive models that consider the overall homogenized material as nonlinear orthotropic. This study examines both approaches for nonlinear modeling of pultruded composites. These micromodels are used for pultruded plates made from alternating roving and CFM layers under general in-plane loading. Nonlinear 3D micromechanical models are formulated for the roving and the CFM layers. An overall plane-stress condition is added as a constraint to the combined set of $3 \mathrm{D}$ micromechanical and constitutive equations of the two micromodels.
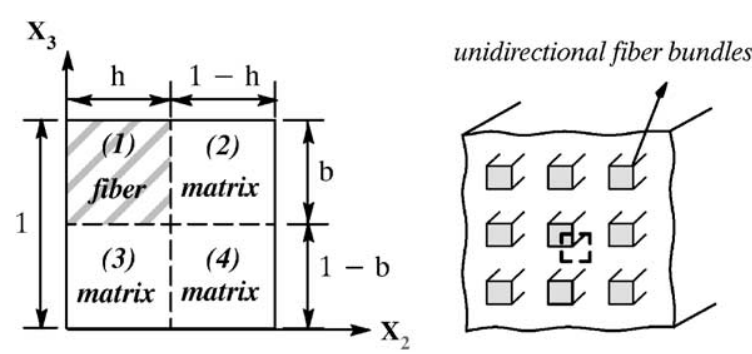

Fig. 1. Roving UC.

A 3D nonlinear micromechanical model for the roving layers is developed based on a rectangular unit-cell (UC) model with four sub-cells used for a unidirectional fiber reinforced material. This model is used to idealize the roving layer as a periodic medium with arrays of fibers having a square section. Fig. 1 describes the idealized periodic medium along with the UC geometry. The roving micromodel is derived by writing approximate traction and displacement continuity relations in terms of average stresses and strains in the sub-cells. This micromechanical model yields comparable results to those generated from the method of cells that was proposed by Aboudi (1991).

The long fibers are aligned in the $x_{1}$ direction. The other cross-section directions are referred to as the transverse directions. The UC is divided into four sub-cells due to symmetry. The traction and displacement continuity relations between the subcells are approximated in terms of the appropriate components of the average stress $\left(\sigma^{(\alpha)}, \alpha=1,2\right.$, $3,4)$ and strain $\left(\varepsilon^{(\alpha)}, \alpha=1,2,3,4\right)$ vectors in the sub-cells. The overall average stress and strain vectors for the UC are denoted by $(\bar{\sigma})$ and $(\bar{\varepsilon})$, respectively.

The notations for the stress and strain vectors, defined in this section, are:

$$
\begin{gathered}
\left\{\sigma_{i}^{(\alpha)}\right\}^{\mathrm{T}}=\left\{\sigma_{11}, \sigma_{22}, \sigma_{33}, \tau_{12}, \tau_{23}, \sigma_{23}\right\}^{(\alpha)} \\
\left\{\varepsilon_{i}^{(\alpha)}\right\}^{\mathrm{T}}=\left\{\varepsilon_{11}, \varepsilon_{22}, \varepsilon_{33}, \lambda_{12}, \lambda_{13}, \lambda_{23}\right\}^{(\alpha)} \\
i=1, \ldots, 6 ; \quad \alpha=1, \ldots, 4
\end{gathered}
$$

where $(\alpha)$ denotes the sub-cell number in the UC and (i) denotes the stress or strain component. 
These are also referred herein as mode-i. The total volume of the UC is taken to be equal to one. The volumes of the four sub-cells are:

$$
\begin{aligned}
& v_{1}=h b ; \quad v_{2}=(1-h) b \\
& v_{3}=h(1-b) ; \quad v_{4}=(1-h)(1-b)
\end{aligned}
$$

The axial strains are the same in all the subcells. Therefore, the longitudinal incremental relations (mode-1) are:

$$
\begin{aligned}
& \mathrm{d} \varepsilon_{1}^{(1)}=\mathrm{d} \varepsilon_{1}^{(2)}=\mathrm{d} \varepsilon_{1}^{(3)}=\mathrm{d} \varepsilon_{1}^{(4)}=\mathrm{d} \bar{\varepsilon}_{1}^{(\mathrm{R})} \\
& v_{1} \mathrm{~d} \sigma_{1}^{(1)}+v_{2} \mathrm{~d} \sigma_{1}^{(2)}+v_{3} \mathrm{~d} \sigma_{1}^{(3)}+v_{4} \mathrm{~d} \sigma_{1}^{(4)}=\mathrm{d} \bar{\sigma}_{1}^{(\mathrm{R})}
\end{aligned}
$$

Consideration of the interfaces with normals in the $x_{2}$ direction, yields the traction continuity conditions for in-plane stress components 22 and 12 , respectively. The corresponding strain compatibility conditions for these modes follow from separately considering sub-cells (1) and (2), and sub-cells (3) and (4), respectively. These relations are used to express traction and compatibility relations for the transverse stress and strain components (22), mode-2, and for axial shear (12), mode-4. For the case of direct transverse mode-2, i.e. components (22), the continuity relations between the sub-cells are:

$$
\begin{aligned}
& \mathrm{d} \sigma_{2}^{(1)}=\mathrm{d} \sigma_{2}^{(2)} \\
& \mathrm{d} \sigma_{2}^{(3)}=\mathrm{d} \sigma_{2}^{(4)} \\
& \frac{v_{1}}{v_{1}+v_{2}} \mathrm{~d} \varepsilon_{2}^{(1)}+\frac{v_{2}}{v_{1}+v_{2}} \mathrm{~d} \varepsilon_{2}^{(2)}=\mathrm{d} \bar{\varepsilon}_{2}^{(\mathrm{R})} \\
& \frac{v_{3}}{v_{3}+v_{4}} \mathrm{~d} \varepsilon_{2}^{(3)}+\frac{v_{4}}{v_{3}+v_{4}} \mathrm{~d} \varepsilon_{2}^{(4)}=\mathrm{d} \bar{\varepsilon}_{4}^{(\mathrm{R})}
\end{aligned}
$$

For the in-plane shear (mode-4), the relations are:

$$
\begin{aligned}
& \mathrm{d} \sigma_{4}^{(1)}=\mathrm{d} \sigma_{4}^{(2)} \\
& \mathrm{d} \sigma_{4}^{(3)}=\mathrm{d} \sigma_{4}^{(4)} \\
& \frac{v_{1}}{v_{1}+v_{2}} \mathrm{~d} \varepsilon_{4}^{(1)}+\frac{v_{2}}{v_{1}+v_{2}} \mathrm{~d} \varepsilon_{4}^{(2)}=\mathrm{d} \bar{\varepsilon}_{4}^{(\mathrm{R})} \\
& \frac{v_{3}}{v_{3}+v_{4}} \mathrm{~d} \varepsilon_{4}^{(3)}+\frac{v_{4}}{v_{3}+v_{4}} \mathrm{~d} \varepsilon_{4}^{(4)}=\mathrm{d} \bar{\varepsilon}_{4}^{(\mathrm{R})}
\end{aligned}
$$

Consideration of the interfaces with normals in the $x_{3}$ direction, yields the traction continuity conditions for out-of-plane stress components 33 and 13, respectively. The corresponding strain compatibility conditions for these modes follow from separately considering sub-cells (1) and (3), and sub-cells (2) and (4), respectively. These relations are expressed for the direct stress component 33 (mode-3), as:

$$
\begin{aligned}
& \mathrm{d} \sigma_{3}^{(1)}=\mathrm{d} \sigma_{3}^{(3)} \\
& \mathrm{d} \sigma_{3}^{(2)}=\mathrm{d} \sigma_{3}^{(4)} \\
& \frac{v_{1}}{v_{1}+v_{3}} \mathrm{~d} \varepsilon_{3}^{(1)}+\frac{v_{3}}{v_{1}+v_{3}} \mathrm{~d} \varepsilon_{3}^{(3)}=\mathrm{d} \bar{\varepsilon}_{3}^{(\mathrm{R})} \\
& \frac{v_{2}}{v_{2}+v_{4}} \mathrm{~d} \varepsilon_{3}^{(2)}+\frac{v_{4}}{v_{2}+v_{4}} \mathrm{~d} \varepsilon_{3}^{(4)}=\mathrm{d} \bar{\varepsilon}_{3}^{(\mathrm{R})}
\end{aligned}
$$

For the out-of-plane shear component 13 (mode-5), the relations are:

$$
\begin{aligned}
& \mathrm{d} \sigma_{5}^{(1)}=\mathrm{d} \sigma_{5}^{(3)} \\
& \mathrm{d} \sigma_{5}^{(2)}=\mathrm{d} \sigma_{5}^{(4)} \\
& \frac{v_{1}}{v_{1}+v_{3}} \mathrm{~d} \varepsilon_{5}^{(1)}+\frac{v_{3}}{v_{1}+v_{3}} \mathrm{~d} \varepsilon_{5}^{(3)}=\mathrm{d} \bar{\varepsilon}_{5}^{(\mathrm{R})} \\
& \frac{v_{2}}{v_{2}+v_{4}} \mathrm{~d} \varepsilon_{5}^{(2)}+\frac{v_{4}}{v_{2}+v_{4}} \mathrm{~d} \varepsilon_{5}^{(4)}=\mathrm{d} \bar{\varepsilon}_{5}^{(\mathrm{R})}
\end{aligned}
$$

Finally, in the transverse shear mode, mode-6, the traction continuity at all the interfaces between the sub-cells must be satisfied. Since this relation is satisfied using the sub-cells' average stress, the traction continuity and compatibility equations for the transverse shear are:

$$
\begin{aligned}
& \mathrm{d} \sigma_{6}^{(1)}=\mathrm{d} \sigma_{6}^{(2)}=\mathrm{d} \sigma_{6}^{(3)}=\mathrm{d} \sigma_{6}^{(4)} \\
& v_{1} \mathrm{~d} \varepsilon_{6}^{(1)}+v_{2} \mathrm{~d} \varepsilon_{6}^{(2)}+v_{3} \mathrm{~d} \varepsilon_{6}^{(3)}+v_{4} \mathrm{~d} \varepsilon_{6}^{(4)}=\mathrm{d} \bar{\varepsilon}_{6}^{(\mathrm{R})}
\end{aligned}
$$

Eqs. (2)-(8) completely define the micromechanical relations between the stresses and the strains in the sub-cells and the overall average stresses and strains of the roving. These relations are used in incremental (rate) form because the constitutive relations in the matrix sub-cells are nonlinear.

A simplified phenomenological model is proposed for the CFM medium using weighted responses of a unidirectional layer in both axial and transverse type modes. The CFM layer is a medium where resin is reinforced with several mats of relatively long swirl filaments. The fibers are randomly distributed in the plane of the mat. The proposed CFM micromodel generates the overall effective nonlinear 3D response from average 
responses of the two unidirectional layers with axial and transverse fiber orientations. The overall in-plane average stress response is generated by averaging the in-plane stress responses of the two layers while the in-plane strain is the same in all sub-cells. The FVF in the CFM is used to define the relative thicknesses of the two layers. The overall out-of-plane response is generated using traction continuity between the two layers. The CFM effective medium should be represented with in-plane isotropic model. The current model does satisfy this requirement when the fiber is isotropic. In the case where the fiber is orthotropic, the resulting effective properties should be integrated and averaged in the radial direction.

The CFM UC model is shown in Fig. 2 as a collection of four sub-cells. It is also convenient to divide the sub-cells into two parts. The matrixmode layer (part-A) is composed of sub-cells (1) and (2), while the fiber-mode layer (part-B) is composed of sub-cells (3) and (4). The relative thickness of each layer is defined using the FVF, as shown in Fig. 2. The out-of-plane direction is represented by the $x_{3}$ axis. The formulation of the CFM can be presented in terms of average stresses and strains in sub-cells $\mathrm{A}$ and $\mathrm{B}$ as intermediate variables. Therefore, these two parts or sub-cells can be considered in the CFM formulation as two independent layers. The FVFs within the two parts are the same and provide the relations:

$\frac{V_{1}}{V_{1}+V_{2}}=h=v_{\mathrm{fc}} ; \quad \frac{V_{4}}{V_{3}+V_{4}}=\xi=v_{\mathrm{fc}}$

The out-of-plane traction continuity and interface displacement continuity, between parts- $A$ and $\mathrm{B}$, are expressed by:

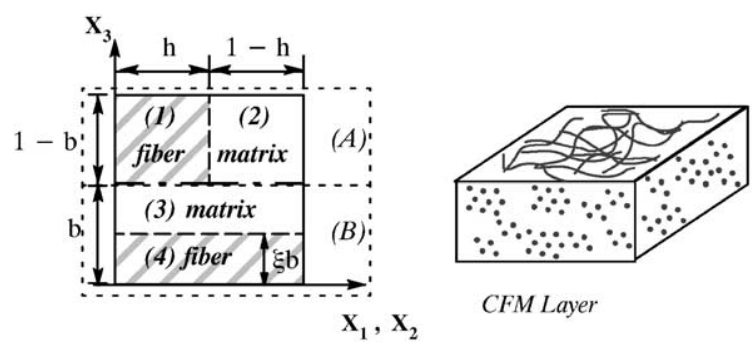

Fig. 2. CFM UC. $\mathrm{d} \bar{\sigma}_{0}^{(\mathrm{C})}=\mathrm{d} \sigma_{0}^{(\mathrm{A})}=\mathrm{d} \sigma_{0}^{(\mathrm{B})}$

$\mathrm{d} \bar{\varepsilon}_{i}^{(\mathrm{C})}=\mathrm{d} \varepsilon_{i}^{(\mathrm{A})}=\mathrm{d} \varepsilon_{i}^{(\mathrm{B})}$

where a CFM quantity is denoted by a (C) superscript and an overbar is used to denote an averaged variable. The homogenized in-plane stresses and out-of-plane strains are taken as weighted averages, using the FVF in the CFM, as:

$\mathrm{d} \bar{\sigma}_{i}^{(\mathrm{C})}=\frac{1}{V}\left(V_{\mathrm{A}} \mathrm{d} \sigma_{i}^{(\mathrm{A})}+V_{\mathrm{B}} \mathrm{d} \sigma_{i}^{(\mathrm{B})}\right)$
$\mathrm{d} \bar{\varepsilon}_{0}^{(\mathrm{C})}=\frac{1}{V}\left(V_{\mathrm{A}} \mathrm{d} \varepsilon_{0}^{(\mathrm{A})}+V_{\mathrm{B}} \mathrm{d} \varepsilon_{0}^{(\mathrm{B})}\right)$

Within the matrix-mode layer (part-A), the following relations for all stress and strain components should be satisfied:

$\mathrm{d} \bar{\sigma}^{(\mathrm{A})}=\mathrm{d} \sigma^{(1)}=\mathrm{d} \sigma^{(2)}$

$\mathrm{d} \bar{\varepsilon}^{(\mathrm{A})}=\frac{1}{V_{\mathrm{A}}}\left(V_{1} \mathrm{~d} \varepsilon^{(1)}+V_{2} \mathrm{~d} \varepsilon^{(2)}\right)$

The corresponding equations for the fiber-mode layer (part-B) are:

$\mathrm{d} \bar{\sigma}_{0}^{(\mathrm{B})}=\mathrm{d} \bar{\sigma}_{0}^{(3)}=\mathrm{d} \bar{\sigma}_{0}^{(4)}$

$\mathrm{d} \bar{\varepsilon}_{i}^{(\mathrm{B})}=\mathrm{d} \varepsilon_{i}^{(3)}=\mathrm{d} \varepsilon_{i}^{(4)}$

$\mathrm{d} \bar{\sigma}_{i}^{(\mathrm{B})}=\frac{1}{V_{\mathrm{B}}}\left(V_{3} \mathrm{~d} \sigma_{i}^{(3)}+V_{4} \mathrm{~d} \sigma_{i}^{(4)}\right)$

$\mathrm{d} \bar{\varepsilon}_{0}^{(\mathrm{B})}=\frac{1}{V_{\mathrm{B}}}\left(V_{3} \mathrm{~d} \varepsilon_{0}^{(3)}+V_{4} \mathrm{~d} \varepsilon_{0}^{(4)}\right)$

Eqs. (9)-(13) define the 3D micromechanical relations between the average stresses and strains in the fiber and matrix sub-cells of the CFM layer. These relations are used in an incremental form because the constitutive relations in the two matrix sub-cells are nonlinear. The linearized solution, in the form of stresses and strains in the sub-cells, is obtained from the incremental equations of both CFM and roving micromodels. This is a trial state solution that usually violates the nonlinear constitutive relations. Once the constitutive relations are satisfied and the correct state of stress is obtained for the trial incremental strain, some traction continuity and strain compatibility equations are then violated. These are used to form a residual vector expressed using total stress and strain quantities. The next step is to obtain a correction 
to the trial state that minimizes the residual vector. This process is repeated, i.e. calculations of the constitutive and micromechanical relations, until the converged solution satisfies both sets of equations numerically.

\section{Calibration of the micromodels}

The in situ linear and nonlinear material properties for the fiber and matrix constituents in the roving and the CFM micromodels are assumed to be the same. These properties are calibrated in two steps. First, the fiber constituent is assumed linear elastic with stiffness values usually available in the literature. In the current study, the material have the same E-glass fiber in both the roving and CFM; the fiber properties are listed in Table 1. However, determining the linear elastic properties for the matrix constituent is not a straight forward and there is not a unique method to achieve that. In fact, the matrix medium is a randomly heterogeneous medium that includes polymeric matrix and other additives, such as clay or microspheres. In addition, the existence of voids and microcracks, due to manufacturing is also lumped in the effective property of this isotropic matrix phase. The nonlinear material response of the composite is attributed to the matrix and it is modeled using the $\mathbf{J} 2$ deformation theory along with the Ramberg-Osgood nonlinear uniaxial representation. It should be mentioned that the matrix sub-cells, in both the roving and CFM micromodels, are assumed to have the same linear and nonlinear parameters. The V-notch shear test is used to calibrate the nonlinear matrix parameters. Three repeated V-notch tests were conducted in this study and they are used to determine the in situ Ramberg-Osgood nonlinear shear stress-strain

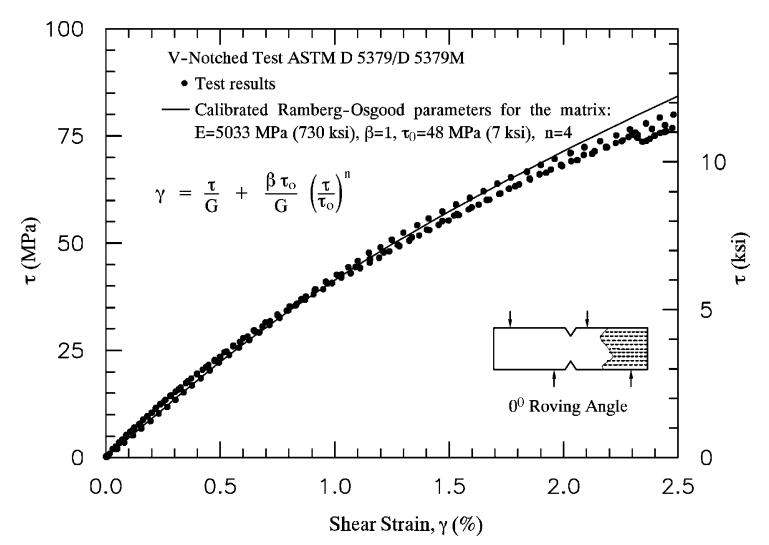

Fig. 3. Shear response of E-glass/vinylester composite used to calibrate the in situ Ramberg-Osgood equation for the matrix.

curve, as shown in Fig. 3. The three RambergOsgood parameters for the nonlinear matrix response along with the linear elastic properties of the fiber and the matrix complete the set of properties needed for the proposed micromechanical models. All linear and nonlinear properties are listed in Table 1.

\section{Nonlinear anisotropic macromodels for pultruded composites}

The purpose of this section is to apply existing nonlinear orthotropic material models for the multi-axial response of pultruded composites. Two previously developed nonlinear orthotropic macromodels are proposed for pultruded composites under a state of plane stress. These models were mainly used for laminated composites. The first model is the Hahn and Tsai (1973) nonlinear model for orthotropic lamina under a state of plane stress. This model considers the nonlinear response to be solely dependent on the axial-shear

Table 1

Fiber and matrix linear and nonlinear elastic properties used in the $3 \mathrm{D}$ micromodels for roving and CFM (FVF in: roving layers $=0.407, \mathrm{CFM}$ layers $=0.305$ )

\begin{tabular}{llllll}
\hline & \multicolumn{2}{l}{ (Ramberg-Osgood) } & & \\
\cline { 2 - 6 } & $E(1000 \mathrm{ksi})$ & $v(1000 \mathrm{ksi})$ & $\beta(\mathrm{ksi})$ & $\tau_{0}(\mathrm{ksi})$ & \\
\hline Fiber (E-glass) (isotropic) & 10.5 & 0.25 & & 4.0 & 7.0 \\
Matrix (vinylester + fillers) (isotropic) & 0.730 & 0.30 & 1 & & 7 \\
\hline
\end{tabular}


stress. The only nonlinear strain component is also the axial shear. As a result, the complementary energy function is composed of a quadratic polynomial, representing linear stress-strain relations, and a fourth order term in the shear stress representing the nonlinear inelastic shear strain. The nonlinear parameters can be obtained by the calibration of axial shear test data with the Ramberg-Osgood ( $\mathrm{R}-\mathrm{O})$ parametric curve. The total strain-stress relation can be expressed for a unidirectional lamina as:

$$
\begin{aligned}
\left\{\begin{array}{l}
\varepsilon_{11} \\
\varepsilon_{22} \\
\gamma_{12}
\end{array}\right\}= & {\left[\begin{array}{ccc}
\frac{1}{E_{11}} & -\frac{v_{12}}{E_{11}} & 0 \\
& \frac{1}{E_{22}} & 0 \\
\text { symm. } & & \frac{1}{G_{12}}
\end{array}\right]\left\{\begin{array}{l}
\sigma_{11} \\
\sigma_{22} \\
\tau_{12}
\end{array}\right\} } \\
& +\left[\begin{array}{ccc}
0 & 0 & 0 \\
0 & 0 & 0 \\
0 & 0 & S_{66}^{\prime}
\end{array}\right]\left\{\begin{array}{l}
\sigma_{11} \\
\sigma_{22} \\
\tau_{12}
\end{array}\right\}
\end{aligned}
$$

where the additional compliance term for shear is:

$S_{66}^{\prime}=\frac{\beta}{G_{12}}\left(\frac{\tau_{12}}{\tau_{0}}\right)^{n-1}$

and the $\tau_{0}, \beta$, and $n$ are material parameters obtained from the in-plane shear stress-strain of the material. The above Ramberg-Osgood axial-shear response is calibrated in this study using the Vnotch shear test results. Fig. 4 shows the experimental results along with the fitted curve. The linear elastic properties and nonlinear material parameters used in Eq. (15), for the tested E-glass/ vinylester material, are shown in Table 2. The

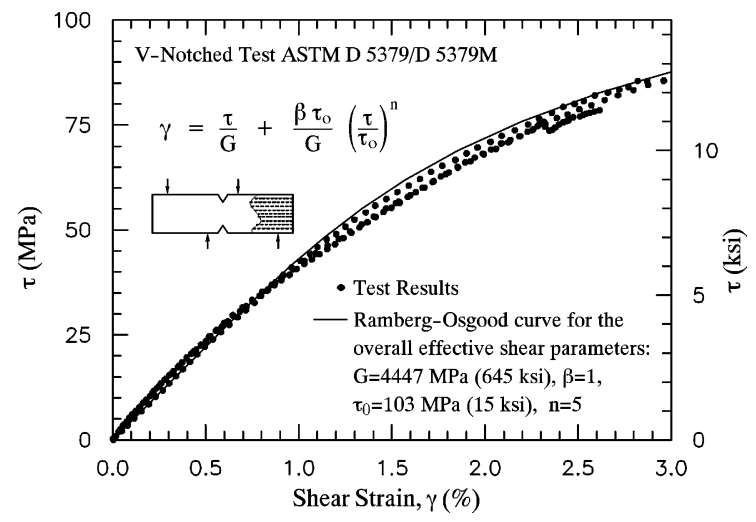

Fig. 4. Ramberg-Osgood curve for the axial-shear stress-strain test data.
Table 2

Effective properties used in the Hahn and Tsai material model

\begin{tabular}{lllllll}
\hline $\begin{array}{l}E_{11} \\
(\mathrm{ksi})\end{array}$ & $\begin{array}{l}E_{22} \\
(\mathrm{ksi})\end{array}$ & $\begin{array}{l}G_{12} \\
(\mathrm{ksi})\end{array}$ & $\begin{array}{l}v_{12} \\
(\mathrm{ksi})\end{array}$ & $\begin{array}{l}\beta \\
(\mathrm{ksi})\end{array}$ & $\begin{array}{l}n \\
(\mathrm{ksi})\end{array}$ & $\begin{array}{l}\tau_{0} \\
(\mathrm{ksi})\end{array}$ \\
\hline 2800 & 1838 & 645 & 0.30 & 1.0 & 5.0 & 15.0 \\
\hline
\end{tabular}

longitudinal, transverse, and shear initial elastic moduli are determined from the experimental compression results calculated according to ASTM standards, Haj-Ali and Kilic (2002). The calibration was performed by using polynomial regression in which the values of $\tau_{0}$ and $n$ were obtained for the best curve fit.

The second nonlinear orthotropic model used in this study was derived by Hashin et al. (1974). This constitutive model is used for a transversely isotropic medium under a state of plane stress. This model assumes that nonlinear inelastic strains exist only in the transverse and axial shear. The longitudinal response is assumed to be linear. The inelastic part of the strains is assumed to depend on quadratic functions in terms of both the axial shear and transverse stresses. These inelastic strains are defined by general quadratic stress functions multiplied by the corresponding deviatoric stress component. The quadratic terms used involve the stress invariants of a medium with transversely isotropic symmetry. The coefficients and power of these functions are obtained by comparing the general form of the inelastic strain components to the corresponding one-dimensional $\mathrm{R}-\mathrm{O}$ representations, separately for axial-shear and transverse compression modes. The total stress-strain relation for a lamina can be expressed as:

$$
\begin{aligned}
\left\{\begin{array}{l}
\varepsilon_{11} \\
\varepsilon_{22} \\
\gamma_{12}
\end{array}\right\}= & {\left[\begin{array}{ccc}
\frac{1}{E_{11}} & -\frac{v_{12}}{E_{11}} & 0 \\
& \frac{1}{E_{22}} & 0 \\
\text { symm. } & & \frac{1}{G_{12}}
\end{array}\right]\left\{\begin{array}{l}
\sigma_{11} \\
\sigma_{22} \\
\tau_{12}
\end{array}\right\} } \\
& +\left[\begin{array}{ccc}
0 & 0 & 0 \\
0 & S_{22}^{\prime} & 0 \\
0 & 0 & S_{66}^{\prime}
\end{array}\right]\left\{\begin{array}{l}
\sigma_{11} \\
\sigma_{22} \\
\tau_{12}
\end{array}\right\}
\end{aligned}
$$

where the above nonlinear inelastic terms are expressed as,

$S_{22}^{\prime}=\frac{1}{E_{22}}\left[\left(\frac{\sigma_{22}}{\sigma_{y}}\right)^{2}+\left(\frac{\tau_{12}}{\tau_{y}}\right)^{2}\right]^{\frac{(m-1)}{2}}$ 


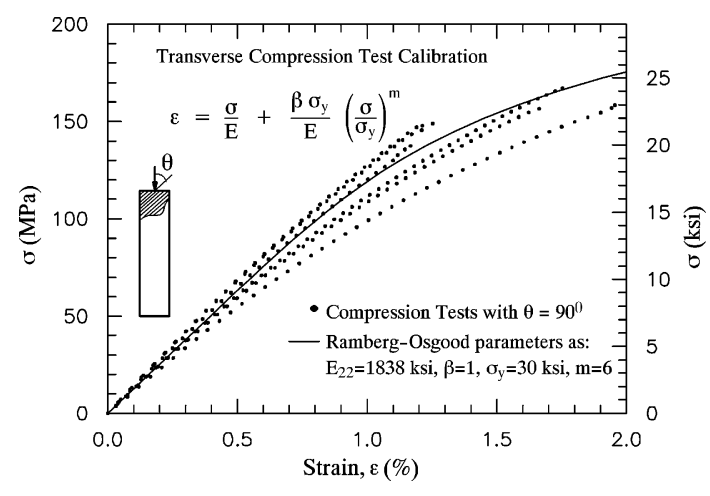

Fig. 5. Ramberg-Osgood curve for the transverse compression stress-strain test data.

Table 3

Effective properties used in the Hashin et al. material model

\begin{tabular}{llllllll}
\hline $\begin{array}{l}E_{11} \\
(\mathrm{ksi})\end{array}$ & $\begin{array}{l}E_{22} \\
(\mathrm{ksi})\end{array}$ & $\begin{array}{l}G_{12} \\
(\mathrm{ksi})\end{array}$ & $\begin{array}{l}v_{12} \\
(\mathrm{ksi})\end{array}$ & $\begin{array}{l}m \\
(\mathrm{ksi})\end{array}$ & $\begin{array}{l}n \\
(\mathrm{ksi})\end{array}$ & $\begin{array}{l}\tau_{y} \\
(\mathrm{ksi})\end{array}$ & $\begin{array}{l}\sigma_{y} \\
(\mathrm{ksi})\end{array}$ \\
\hline 2800 & 1838 & 645 & 0.30 & 6.0 & 5.0 & 15.0 & 30.0 \\
\hline
\end{tabular}

$S_{66}^{\prime}=\frac{1}{G_{12}}\left[\left(\frac{\sigma_{22}}{\sigma_{y}}\right)^{2}\left(\frac{\tau_{12}}{\tau_{y}}\right)^{2}\right]^{\frac{(n-1)}{2}}$

The Ramberg-Osgood parameters in Eq. (17), $\sigma_{y}$ and $m$, are obtained from the calibration with the experimental transverse stress-strain curve in compression. The compression stress-strain test results, generated from transverse pultruded coupons, along with the fitted $\mathrm{R}-\mathrm{O}$ uniaxial curve are shown in Fig. 5. The other Ramberg-Osgood parameters in Eq. (18), $\tau_{y}$ and $n$, are obtained from in-plane shear response in the same manner used for the Hahn and Tsai model, Fig. 3. The linear and nonlinear material properties used in the Hashin et al. model, for E-glass/vinylester pultruded material, are presented in Table 3. The longitudinal, transverse, and shear moduli are the average values of 3-5 repeated tests and are calculated according to ASTM standards.

\section{Nonlinear off-axis stress-strain response}

The prediction capability of the three proposed nonlinear models for pultruded composites is ex- amined for off-axis coupons that are subject to compressive loading. The objective of the off-axis tests is to generate the nonlinear stress-strain behavior up to ultimate failure. Coupons were cut from a pultruded plate such that the roving fibers are oriented at a specified off-axis angle. The tested coupons have the following off-axis angles: $0^{\circ}, 15^{\circ}$, $30^{\circ}, 45^{\circ}, 60^{\circ}$, and $90^{\circ}$. A detailed information on the off-axis tests and the geometry of the coupons can be found in Haj-Ali and Kilic (2002). Each nonlinear off-axis test was repeated 3-5 times.

Figs. 6-11 show the experimental results (with repeated tests) and the predictions of both the proposed 3D micromodel and the anisotropic models. The proposed $3 \mathrm{D}$ micromodel predicts a

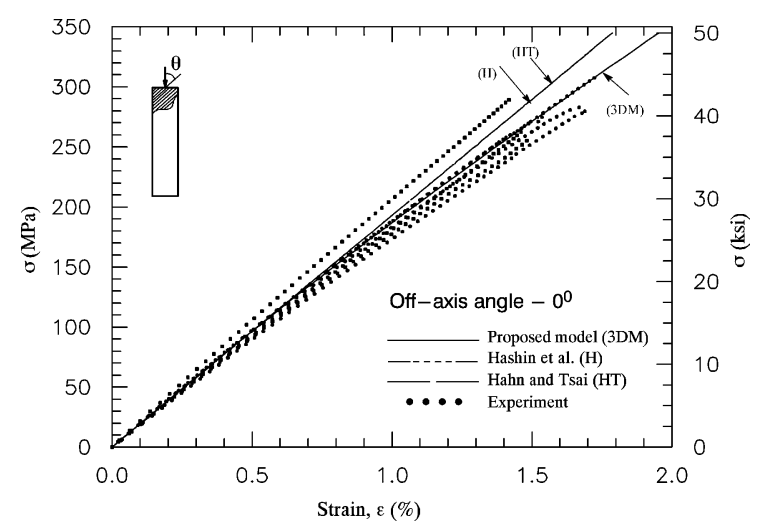

Fig. 6. Stress-strain response from three material models for off-axis $\theta=0^{\circ}$ pultruded coupon under compression.

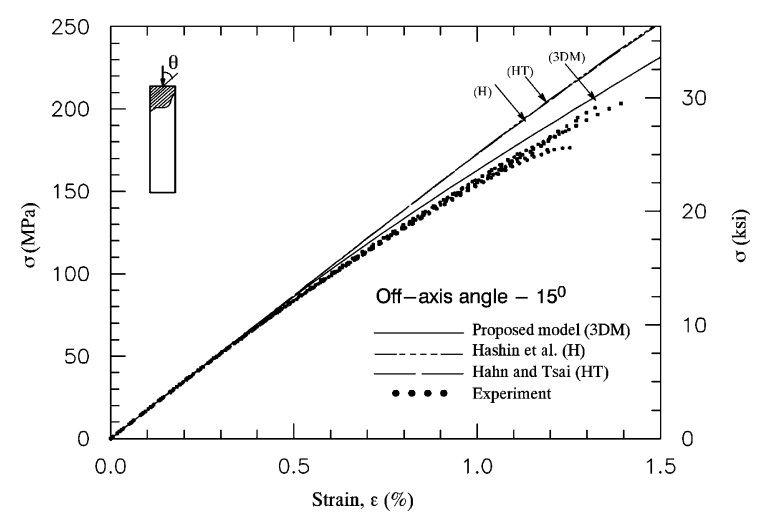

Fig. 7. Stress-strain response from three material models for off-axis $\theta=15^{\circ}$ pultruded coupon under compression. 


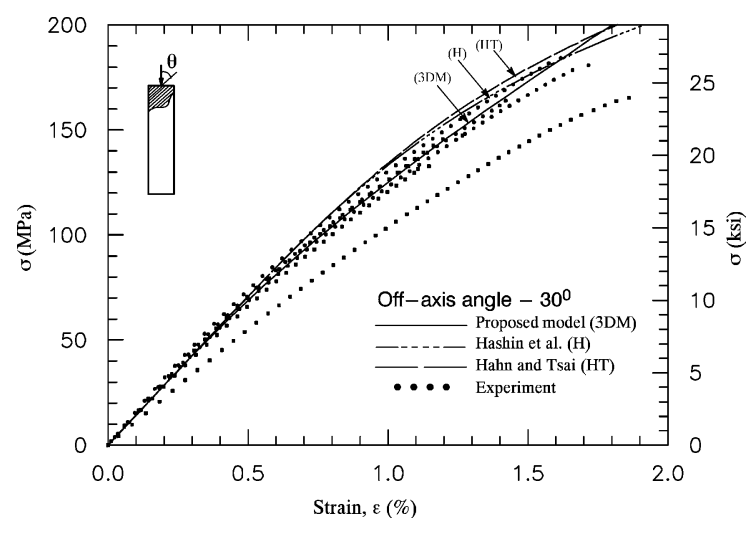

Fig. 8. Stress-strain response from three material models for off-axis $\theta=30^{\circ}$ pultruded coupon under compression.

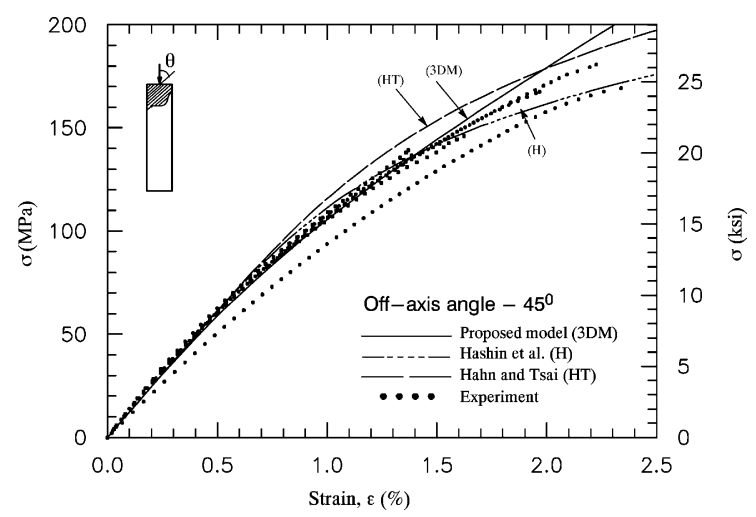

Fig. 9. Stress-strain response from three material models for off-axis $\theta=45^{\circ}$ pultruded coupon under compression.

close response to the overall experimental results. Hahn and Tsai model predicts a linear stressstrain response in the case of $0^{\circ}$ and $90^{\circ}$ orientations. This linear behavior is expected because the nonlinear effects in this model are only due to axial shear stress. The Hashin et al. material model captures well the nonlinear response, but it shows a softer nonlinear behavior at higher strain magnitudes for off-axis angles larger than $30^{\circ}$. It is interesting to note the prediction from the $3 \mathrm{D}$ micromodel for the $45^{\circ}$ off-axis coupons deviates from the experimental results for axial strains larger than $1.5 \%$. The calibration of the $3 \mathrm{D}$ micromodel is in a good agreement with the V-notch effective shear results up to $2.5 \%$ strain, as shown

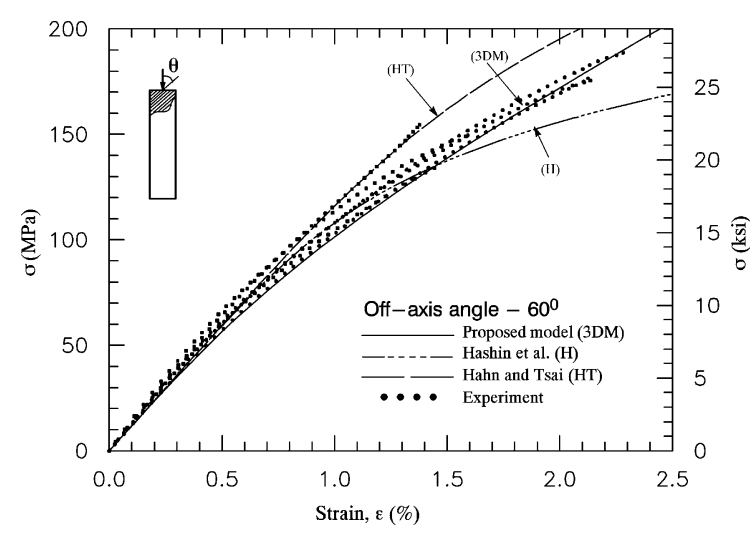

Fig. 10. Stress-strain response from three material models for off-axis $\theta=60^{\circ}$ pultruded coupon under compression.

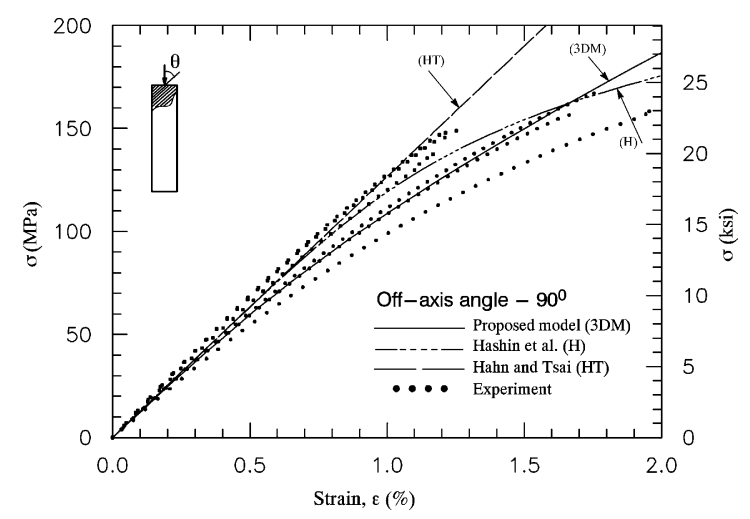

Fig. 11. Stress-strain response from three material models for off-axis $\theta=90^{\circ}$ pultruded coupon under compression.

in Fig. 3. However, both macromodels were successfully calibrated with the same shear results up to $3.0 \%$ strain. This can explain why the micromodel did not perform as well as in the $45^{\circ}$ case beyond $1.5 \%$. Despite this shortcoming, the overall performance of the proposed micromodels is superior when considering the complete response range for all tested angles.

\section{Conclusions}

New 3D micromodels are derived for the nonlinear analysis of pultruded FRP composite materials. The nonlinear response of pultruded FRP composites is examined both experimentally and analytically by using different off-axis coupons in 
compression in order to predict the nonlinear response under different multi-axial stress states. The micromechanical models explicitly recognize the response of the roving and CFM composite systems (layers) and their fiber-matrix constituents within the cross-section of the pultruded member. The nonlinear orthotropic models of Hahn and Tsai, and Hashin et al. are also calibrated and applied to model the multi-axial response of the pultruded coupons. A good agreement is observed between the experimentally obtained material behavior and the proposed micromodels for all off-axis coupons. The nonlinear responses obtained from the two orthotropic models are in good agreement but not for all the tested off-axis coupons.

\section{Acknowledgement}

This work was supported by NSF through the Civil and Mechanical Systems (CMS) Division under grant number 9876080 .

\section{References}

Abu-Farsakh, G., 1989. New material models for nonlinear stress-strain behaviour of composite materials. Composites 20,349 .

Aboudi, J., 1991. Mechanics of composite materials - a unified micromechanical approach. Elsevier, Amsterdam.

Amijima, S., Adachi, T., 1979. Nonlinear stress-strain response of laminated composites. J. Compos. Mater. 13, 206-218.

Binshan, S.Y., Svenson, A.L., Bank, L.C., 1995. Mass and volume fraction properties of pultruded glass fibre composites. Composites 26, 725-731.

Dvorak, G.J., Rao, M.S., 1976. Axisymmetric plasticity theory of fibrous composites. Int. J. Eng. Sci. 14, 361-373.

Griffin, O.H., Kamat, M.P., Herakovich, C.T., 1981. Threedimensional finite element analysis of laminated composites. J. Composite Materials 5, 543-560.

Haj-Ali, R.M., Kilic, H., Zureick, A.-H., 2001. Three-dimensional micromechanics-based constitutive framework for analysis of pultruded composite structures. J. Eng. Mech. $127,1-8$.
Haj-Ali, R.M., Kilic, H. Nonlinear behavior of pultruded FRP composites. Composites: Part B, Engineering 33, 173-191.

Hahn, T.H., Tsai, S.W., 1973. Nonlinear elastic behavior of unidirectional composite laminae. J. Compos. Mater. 7, 102-118.

Hahn, T.H., 1973. Nonlinear behaviour of laminated composites. J. Compos. Mater. 7, 257-271.

Hashin, Z., Bagchi, D., Rosen, B.W., 1974. Nonlinear behavior of fiber composite laminates, NASACR-2313.

Herakovich, C.T., Mirzadeh, F., 1991. Properties of pultruded graphite/epoxy. J. Reinf. Plast. Comp. 10, 2-28.

Jones, R.M., Nelson Jr., D.A.R., 1974. A new material model for the nonlinear biaxial behavior of ATJ-S graphite. J. Compos. Mater. 9, 10-27.

Jones, R.M., Morgan, H.S., 1977. Analysis of nonlinear stressstrain behavior of fiber reinforced materials. AIAA J. 15, 1669-1676.

Kuppuswamy, T., Nada, A., Reddy, J.N., 1984. Materially nonlinear analysis of laminated composite plates. Compos. Struct. 2, 315-328.

Luciano, R., Barbero, E.J., 1994. Formulae for the stiffness of composites with periodic micro-structure. Int. J. Solids Struct. 31 (21), 2933-2944.

Mathison, S.R., Pindera, M.J., Herakovich, C.T., 1991. Nonlinear response of resin matrix laminates using endochronic theory. J. Eng. Mat. Tech. 113, 449-455.

Nahas, M.N., 1984. Analysis of non-linear stress-strain response of laminated fibre-reinforced composites. Fibre Sci. Tech. 20, 297-313.

Petit, P.H., Waddoups, M.E., 1969. A method of predicting the nonlinear behavior of laminated composites. J. Compos. Mater. 3, 2-19.

Pindera, M.J., Herakovich, C.T., 1983. An endochronic model for the response of unidirectional composites under off-axis tensile load. In: Hashin, Z., Herakovich, C.T. (Eds.), Mechanics of Composite Materials: Recent Advances, Proc. IUTAM Symposium on Mechanics of Composite Materials. Pergamon Press, New York, pp. 367-381.

Richard, R., Abbott, B., 1975. Versatile elastic-plastic stressstrain formula. ASCE J. Eng. Mech. Div., Technical Note 101, 511-515.

Sandhu, R.S., 1976. Non-linear behaviour of unidirectional and angle-ply laminates. J. Aircraft 13, 104-111.

Sun, C.T., Chen, J.L., 1989. A simple flow rule for characterizing nonlinear behavior of fiber composites. J. Compos. Mater. 23, 1009-1020.

Wang, Y., Zureick, A., 1994. Characterization of the longitudinal tensile behavior of pultruded I-shape structural members using coupon specimens. Compos. Struct. 29, 463-472. 